\title{
A Influência do Perfil da Ferramenta e Velocidade de Rotação na Soldagem a Ponto por Fricção e Mistura Mecânica da Liga AA 6181-T4
}

\author{
(The Influence of Tool Geometry and Rotational Speed on Friction Stir Spot Welding of Aluminum Alloy AA- 6181-T4)
}

\author{
Fabiano Dornelles Ramos ${ }^{1}$, Telmo Roberto Strohaecker', Jorge Fernandez dos Santos ${ }^{3}$ \\ ${ }^{1}$ Instituto Federal de Educação, Ciência e Tecnologia do Rio Grande do Sul-Campus Caxias do Sul-Caxias do Sul-RS-Brasil. \\ fabiano.ramos@caxias.ifrs.edu.br \\ ${ }^{2}$ Universidade Federal do Rio Grande do Sul-Departamento de Metalurgia- Laboratório de Metalurgia Física- Porto Alegre-RS- \\ Brasil.telmo@demet.ufrgs.br \\ ${ }^{3}$ Helmholtz-Zentrum Geesthacht Centre for Materials and Coastal Research-Joining Technology Group. jorge.dos.santos@hzg.de
}

\begin{abstract}
Resumo
Soldagem a ponto por fricção é um processo que ocorre no estado sólido com alta eficiência energética, baixo custo de produção além de ser um processo ambientalmente limpo. Estes processos por friç̧ão são uma alternativa a processos convencionais tais como solda ponto por resistência, rebitamento e prensagem a frio. A ausência de uma fase de fusão nesses processos elimina defeitos como porosidades, bolhas, rebaixos, inclusões e microestruturas indesejáveis, que frequentemente aparecem na solda e zonas afetadas pelo calor em processos de soldagem por fusão. O processo de SPFMM consiste em uma ferramenta, que é uma combinação de um pino e um ombro que em rotação, penetra nas chapas formando uma junta sobreposta. O contato do pino com regiões adjacentes e do ombro com a superficie superior da junta geram calor de fricção. Este calor de fricção promove a plastificação do material da junta, que ao mesmo tempo é misturado pelo pino consolidando, assim, a formação da junta. O objetivo do presente trabalho é avaliar a influência da utilização de diferentes perfis de ferramenta e diferentes velocidades de rotação sobre a soldagem e o comportamento mecânico de ligas de alumínio soldadas pelo processo de SPFMM. A caracterização metalúrgica foi feita através de microscopia ótica, lupa e eletrônica de varredura. A caracterização mecânica das juntas foi feita através de ensaios de cisalhamento, perfis de microdureza e monitoramento de torque e força durante o processo de soldagem. Ainda foram realizadas análises do fluxo de material durante o processo. Foi possivel observar uma tendência de aumentar a resistência mecânica da junta com a utilização de velocidades de rotação mais elevadas em ambos os materiais. A utilização de ferramentas com diferentes perfis apresentou diferenças representativas, porém com muita dependência da combinação de velocidade de rotação, pino e ombro utilizados.
\end{abstract}

Palavras-chave: Solda Fricção, Perfil da Ferramenta, Velocidade de Rotação.

Abstract: Friction Stir Spot Welding is a process that occurs in solid state with high energy efficiency, low manufacture costs and environmentally friendly. These friction processes are an alternative to conventional processes like resistance spot welding, riveting and clinching. The absent of a fusion zone on these processes avoid defects like porosity, bubbles, recess, inclusions and undesired microstructures, that appears very often on weld metal and heat affected zones of fusion weld processes. The Friction Stir Spot Welding process (SPFMM- FSSW) consists in a rotating tool (pin and shoulder combined) that penetrates the overlap joint stirring the material and consolidating the joint. The contact of adjacent region of the tool generates the friction heating and, this heating, promotes the plasticization of the material that, at the same time, is stirred by the tool pin leaving a stir zone and a keyhole after process is finished. The aim of the present work is to evaluate the influence of tool profile and rotational speed on the mechanical and metallurgical behavior of friction stir spot welded AA6181-T4 aluminum alloy. The metallurgical characterization was performed through optical microscopy, stereoscope and scanning electron microscopy. The mechanical testing was made trough shear test, microhardness profile and torquelforces monitoring during the process. It was possible to observe a tendency of increasing in mechanical strength of the joint with applying higher rotational speed values. The utilization of different tool profiles presented significant differences, but, with much reliance of the rotational speed, pin and shoulder combination.

Key-words: Friction welding, Tool Profile, Rotational Speed.

\section{Introdução}

Soldagem a ponto por friç̧ão e mistura mecânica é um

(Recebido em 29/02/2012; Texto final em 02/03/2012).

Artigo originalmente publicado no CONSOLDA 2011 técnica relativamente nova (quando comparada a técnicas convencionais de soldagem) onde uma ferramenta rotativa compreendida por um pino e um ombro é penetrada numa junta sobreposta. Este método é aplicável para unir pelo menos duas chapas na posição sobreposta e deixa um furo remanescente ao final do processo. O Instituto alemão HZG (Helmholtz Zentrum Geesthacht) têm estudado esta técnica como um alternativa 

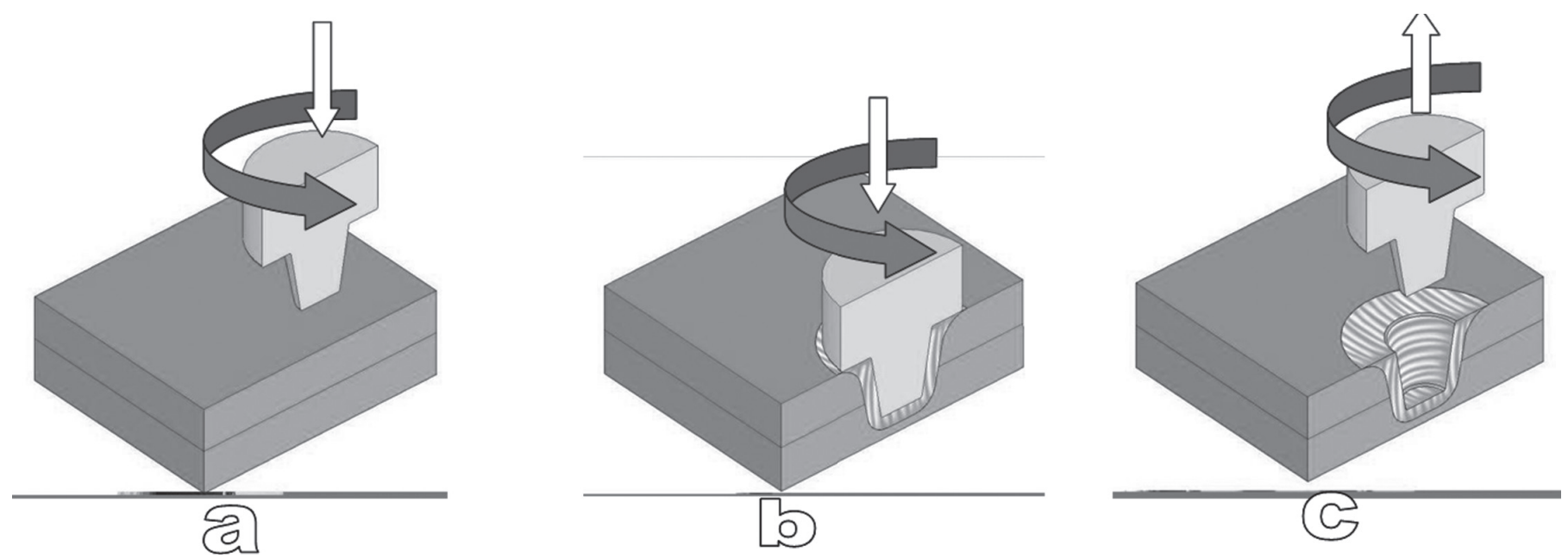

Figura 1- Representação esquemática do processo de SPFMM: a) Penetração da ferramenta b) Mistura do material c) Remoção da ferramenta.

promissora às técnicas convencionais de soldagem a ponto por resistência e rebitamento, desde que combina uma soldagem no estado sólido (evitando todos os problemas relacionados a solidificação do material) com uma junta de alta resistência mecânica e um processo de baixo custo operacional (de acordo com Mazda [24] os custos energéticos podem ser reduzidos em até $99 \%$ quando comparados com a soldagem a ponto por resistência). Como uma variação da soldagem por friç̧ão e mistura mecânica, o processo foi criado e patenteado pelo HZG (antigo GKSS Forschungszentrum)[1-26].

O processo é composto basicamente por três etapas: Penetração da ferramenta, mistura do material e remoção da ferramenta. A primeira etapa consiste na penetração da ferramenta através da junta sobreposta, onde a ferramenta, já em rotação, penetra o pino através da junta. Nesta etapa ocorre um crescimento das forças envolvidas no processo gradualmente até que atinjam um máximo. A segunda etapa ocorre quando o pino já está totalmente penetrado e ocorre o contato do ombro com a chapa superior e o material está sendo misturado (chapa inferior com chapa superior). E a última etapa é a remoção da ferramenta, que ainda em rotação é removida da junta deixando um furo remanescente ao final do processo. As três etapas do processo estão representadas na Figura 1.

O tempo de duração do processo é normalmente bastante curto (entre 1 e 5 segundos envolvendo os três estágios) e a geometria da ferramenta desempenha um papel importantíssimo, já que a maior parte do calor gerado durante o processo provém do contato ombro/chapa superior e o pino que proporciona a mistura e plastificação do material. Muitos trabalhos foram desenvolvidos para avaliar a influência da ferramenta (geometria, percurso, etc) sobre o processo de SPFMM. Na busca de se obter soldas de alta resistência mecânica, percebeu-se que a área soldada é um fator de suma importância para a resistência mecânica da junta. Desta forma, diversos processos foram desenvolvidos com a intenção de realizar soldas com uma maior área de solda. Dentre eles podemos destacar: Stitch FSSW, Squircle FSSW, Swing FSSW and Octaspot FSSW [20, 21, 22].

De acordo com o trabalho desenvolvido por Ramos [1, 26] e Feng [9], a área soldada é considerada uma camada de ligação, ou seja, a área efetiva de solda que suporta os esforços mecânicos da junta na seção transversal da solda. Quanto maior for a camada de ligação, maior será a resistência mecânica da junta soldada.

Após a realização da solda, normalmente quatro diferentes zonas podem ser observadas: A zona de mistura (ZM), a zona termomecanicamente afetada (ZTMA), a zona termicamente afetada (ZTA) e o metal de base (MB). A ZM é uma região composta de grãos pequenos, que tiveram energia termomecânica suficiente para que ocorra a recristalização dos grãos. A ZTMA é composta por grãos retorcidos, que sofreram influência da ferramenta, porém, não tiveram energia suficiente para a recristalização. A ZTA é uma região onde apenas a influência térmica pode ser observada (as vezes ocorre crescimento do grão). E, por fim, a MB que é a região do material original inalterado pelo processo.

O objetivo principal do trabalho é desenvolver as soldas com os parâmetros determinados e verificar a influência dos diferentes perfis de ferramentas e velocidades de rotação sobre as propriedades mecânicas e metalúrgicas das juntas soldadas.

\section{Materiais e Métodos}

\section{MATERIAL}

Foram utilizadas chapas de $1.5 \mathrm{~mm}$ de espessura do material AA 6181 na condição T4. A composição química é mostrada na Tabela 1 (obtida por espectroscopia de emissão ótica).

Tabela 1- Composição química do material base.

\begin{tabular}{|l|l|}
\hline Elemento & \% em peso \\
\hline $\mathrm{Si}$ & 0,85 \\
\hline $\mathrm{Fe}$ & 0,25 \\
\hline $\mathrm{Mn}$ & 0,09 \\
\hline $\mathrm{Mg}$ & 0,74 \\
\hline $\mathrm{Cu}$ & 0,06 \\
\hline $\mathrm{Cr}$ & 0,01 \\
\hline $\mathrm{Ti}$ & 0,003 \\
\hline $\mathrm{Zn}$ & 0,01 \\
\hline $\mathrm{Al}$ & Balanço \\
\hline
\end{tabular}



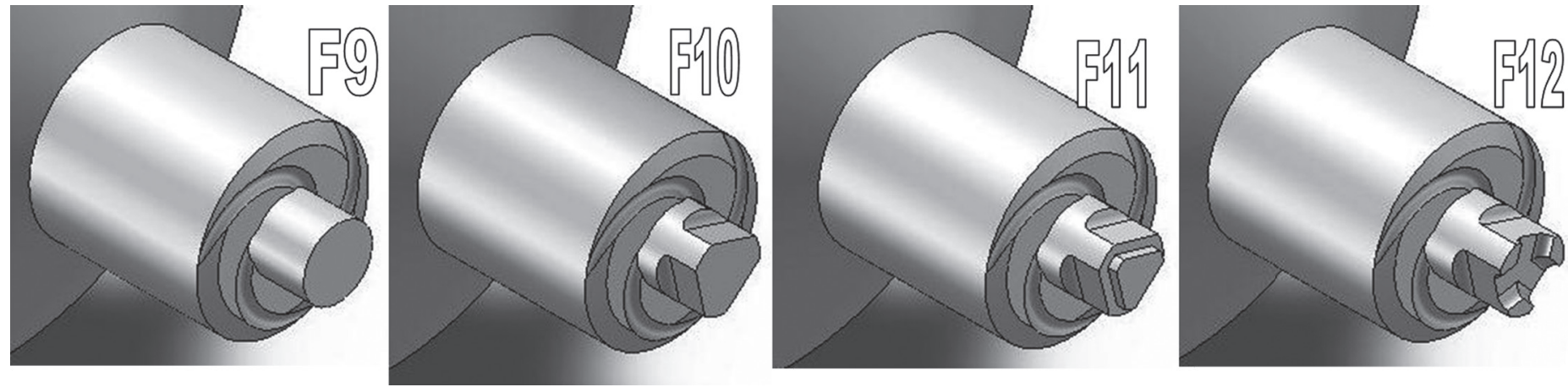

Figura 2- Desenho esquemático representando as ferramentas utilizadas.

\section{SOLDAGEM DAS AMOSTRAS/ ESCOLHA DA} FERRAMENTA

As soldas foram realizadas utilizando-se a variante de controle de deslocamento da máquina chamada Gantry Machine, uma máquina estilo pórtico com capacidade de carga axial e deslocamento horizontal. A máquina trabalha com um sistema de fixação da ferramenta de $20 \mathrm{~mm}$, ou mesmo $15 \mathrm{~mm}$ utilizando adaptador. As velocidades de rotação utilizadas foram de 1000, 2000 e 3000 RPM, com uma ferramenta composta por um ombro com três ranhuras e pino cilíndrico (F9), pino com três superfícies planas (F10), pino com três superfícies planas e um ressalto (F11) e pino com três superfícies planas e três protuberâncias (F12). A Figura 2 mostra o aspecto das quatro ferramentas num desenho esquemático.

As ferramentas tem um diâmetro de $10 \mathrm{~mm}$ no ombro e $4 \mathrm{~mm}$ no pino, com um comprimento de pino de $2 \mathrm{~mm}$. A taxa de penetração utilizada foi de $1 \mathrm{~mm} / \mathrm{s}$ com um tempo de espera (tempo de ferramenta em penetração total) de $2 \mathrm{~s}$ e profundidade de penetração de $2,2 \mathrm{~mm}$. A medição de torque força durante o processo foi realizado pela célula de carga da própria máquina. A literatura diz que velocidades de rotação abaixo de 1000 produzem espessuras muito baixas de camada de ligação, portanto foram escolhidas velocidades de rotação do menor limite(1000 RPM) ao maior limite (3000 RPM- limite da máquina). Para a caracterização do fluxo de material, foram soldadas amostras com uma fina chapa de cobre entre elas $(0,4$ $\mathrm{mm})$ para dar contraste e podermos perceber que direções o fluxo de material seguia nas caracterizações metalográficas. Neste caso foi utilizado uma maior profundidade de penetração (3 mm) e uma velocidade de rotação fixa de 3000 RPM.

\section{CARACTERIZAÇÃO METALÚRGICA}

As amostras foram secionadas e embutidas com resina na sua seção transversal. Procedimentos padronizados de metalografia foram empregados para polimento, lixamento e ataque químico utilizando-se o reagente Barker. As amostras foram observadas em microscopia ótica e imagens digitais foram obtidas.

\section{CARACTERIZAÇÃO MECÂNICA}

Para a caracterização da junta foram realizados ensaios de cisalhamento com amostras soldadas em junta sobreposta de 230x60 mm (46 mm de sobreposição, de acordo com a norma DIN 14273:2002). O equipamento utilizado para realizar os ensaios foi uma máquina de fuso Schenk- Trebel RM100 (100 KN de capacidade) com um controlador Zwick e taxas de deslocamentos entre 1 e $2 \mathrm{~mm} / \mathrm{min}$. Perfis de microdureza foram realizados nas amostras utilizadas para metalografia em duas linhas, uma $1 \mathrm{~mm}$ abaixo da superfície superior e outra $0,75 \mathrm{~mm}$ abaixo da linha de contato entre as chapas soldadas.

\section{Resultados e Discussão}

\section{INVESTIGAÇÃO MICROESTRUTURAL}

A metalografia do material base indicou grãos equiaxiais com partículas de segunda fase dispersas ao longo do material, conforme mostra a Figura 3 e Figura 4.

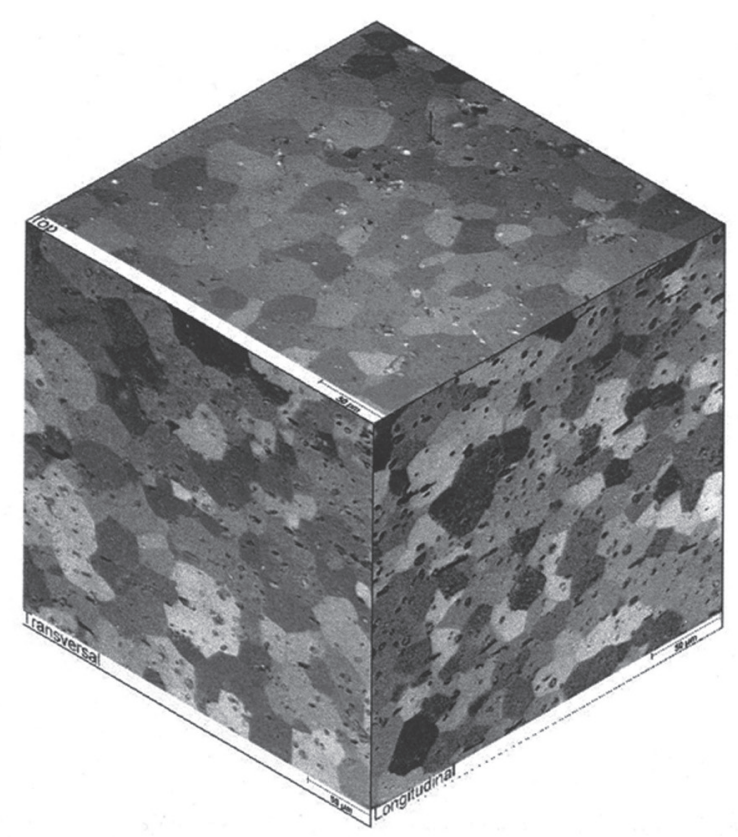

Figura 3- Imagem mostrando a microestrutura original do metal de base da chapa de alumínio AA6181-T4 nas três direções.

A caracterização metalográfica das soldas realizada nas 12 condições indicou a presença de três diferentes zonas: Uma ZM, uma ZTMA e MB (a ZTA não pode ser detectada por esta técnica). A Figura 5 mostra a imagem de uma macrografia realizada na amostra soldada com a ferramenta F11 e velocidade 
de rotação de 2000 RPM. Acima das duas linhas está a ZM, entre as duas linhas está a ZTMA e nas regiões adjacentes a microestrutura inalterada do metal base. As linhas horizontais próximas a região central da junta correspondem a camada de ligação, mostrada por CL.

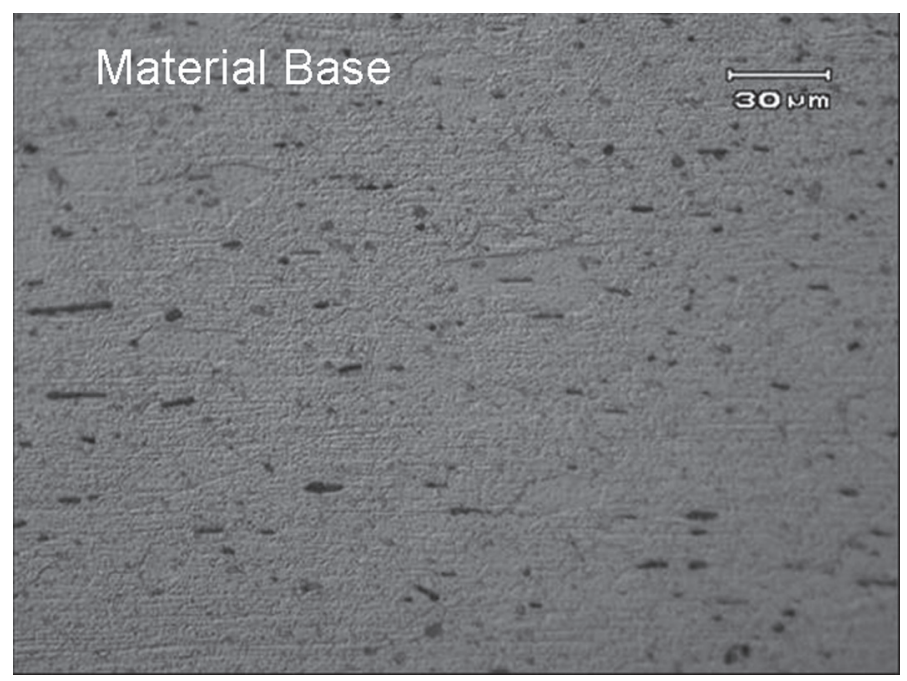

Figura 4- Imagem mostrando a microestrutura do material de base em maior aumento, onde se observa a presença de partículas de segunda fase. mecânica da solda. Este fato se atribui a uma maior seção resistente.

\section{CARACTERIZAÇÃO MECÂNICA}

As amostras ensaiadas em cisalhamento apresentaram três diferentes tipos de modo de falha. A falha por cisalhamento, que envolve baixa deformação plástica, ou seja, uma fratura que envolve menor energia. Uma falha que envolve maior deformação antes da fratura, onde a falha se dá contornando a camada de ligação, ou falha por arrancamento. E a outra é semelhante, também com alta deformação porém ocorre também o destacamento da chapa inferior, ou falha por arrancamento com destacamento da chapa inferior. $\mathrm{O}$ aspecto dos três tipos de falha é mostrado na Figura 8.

A análise da superfície de fratura nos mostra a região da camada de ligação (área efetivamente fraturada e que resistiu a cisalhamento). Esta área foi medida com um paquímetro e inserida em um gráfico juntamente com a resistência final ao cisalhamento das amostras e o que pode ser observado é que a maior resistência da junta se dá quanto maior for a camada de ligação. O resultado dos ensaios de cisalhamento juntamente com os respectivos comprimentos de camada de ligação são apresentados na Figura 9.

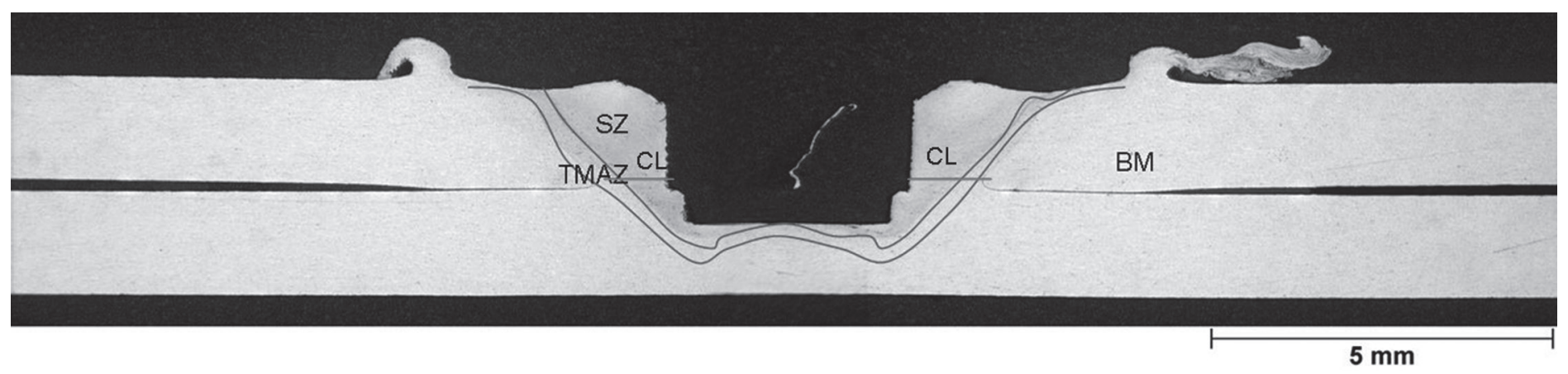

Figura 5- Macrografia onde podem ser observadas as diferentes zonas formadas na solda. TMAZ=ZTMA, $S Z=Z M$ e $B M=M B$.

A microestrutura da ZM e ZTMA ficou semelhante para todas as soldas realizadas, com a ZM apresentando grãos refinados equiaxiais, com partículas de segunda fase redondas e de menor tamanho quando comparadas às observadas no metal de base. Pode-se assumir que a energia de deformação e a ação do pino promoveram a quebra destas partículas em partículas menores. Conforme se observa na Figura 6, que é uma micrografia da região da $\mathrm{ZM}$ da amostra soldada com a ferramenta F11 em 2000 RPM.

Na ZTMA, puderam ser observados grãos alongados e deformados, devido à alta deformação imposta naquela região pela ferramenta, sem a energia térmica suficiente para que houvesse a recristalização, conforme mostra a Figura 7.

A camada de ligação é considerada uma responsável direta pela resistência mecânica da liga, portanto, deve-se buscar sempre as condições que proporcionem a maior camada de ligação possível. De acordo com Ramos e Fheng [1, 9, 26], quanto maior for a camada de ligação, maior será a resistência

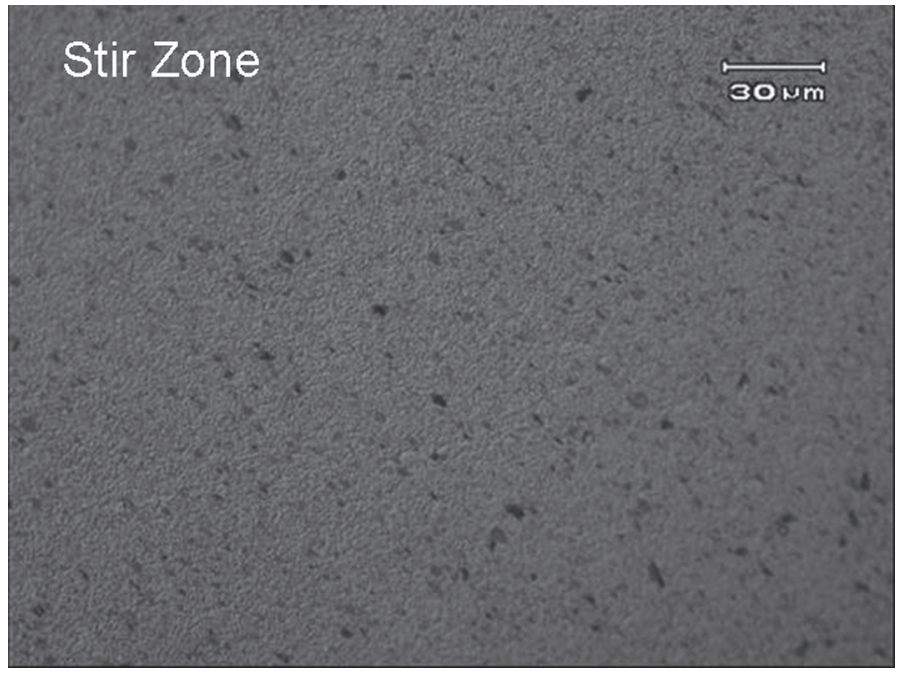

Figura 6- Micrografia da ZM, mostrando o formato das partículas de segunda fase. 


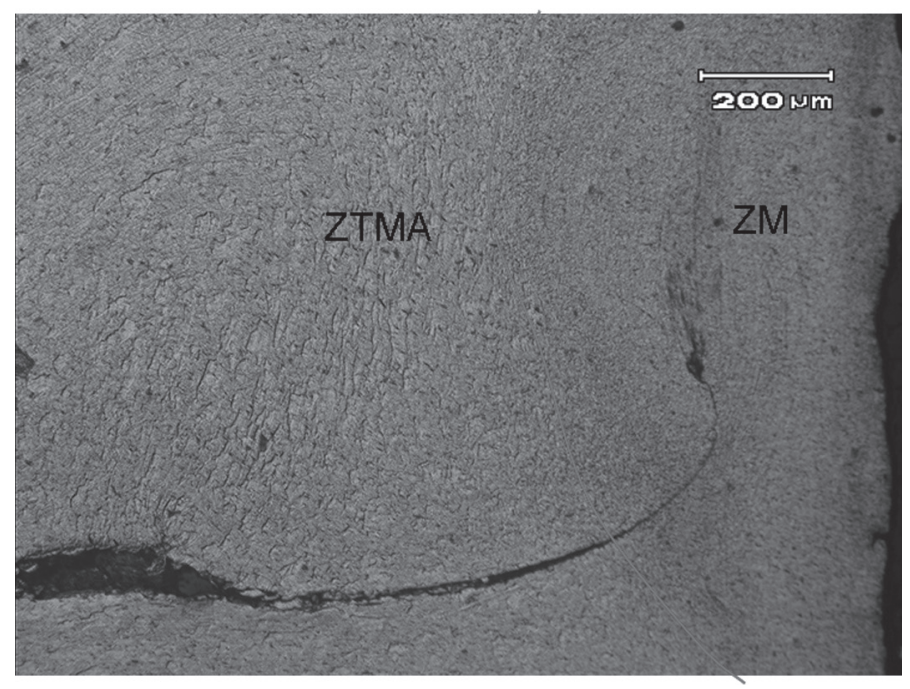

Figura 7- Imagem mostrando a região da ZTMA, onde se observa os grãos alongados deformados. representando o processo.

Como o fluxo de material foi caracterizado apenas com velocidades de rotação de 3000 RPM, a utilização do cobre como traçador não apresenta resultados a respeito da influência da velocidade de rotação sobre o fluxo, o que poderá ser deduzido através da observação das amostras de metalografia.

A ocorrência do modo de falha por cisalhamento implica em menor energia e cargas mais baixas. Desta forma, buscouse uma relação entre a ocorrência de fratura por cisalhamento e o formato obtido na ZM. O que foi encontrado foi que existe um deslocamento da linha de contato das chapas em alguns casos, ou seja, a linha de contato entre as chapas foi deformada e deslocada pra cima. Isso é o que provavelmente influencia na hora do ensaio e evita a ocorrência da fratura por cisalhamento puro, que acarreta em menor deformação e cargas mais baixas de resistência ao cisalhamento. A Figura 11 de Ramos [26] mostra uma amostra soldada em maior velocidade e outra soldada em menor velocidade onde pode ser observado este deslocamento da linha de contato. Ao mesmo tempo em que desloca a linha

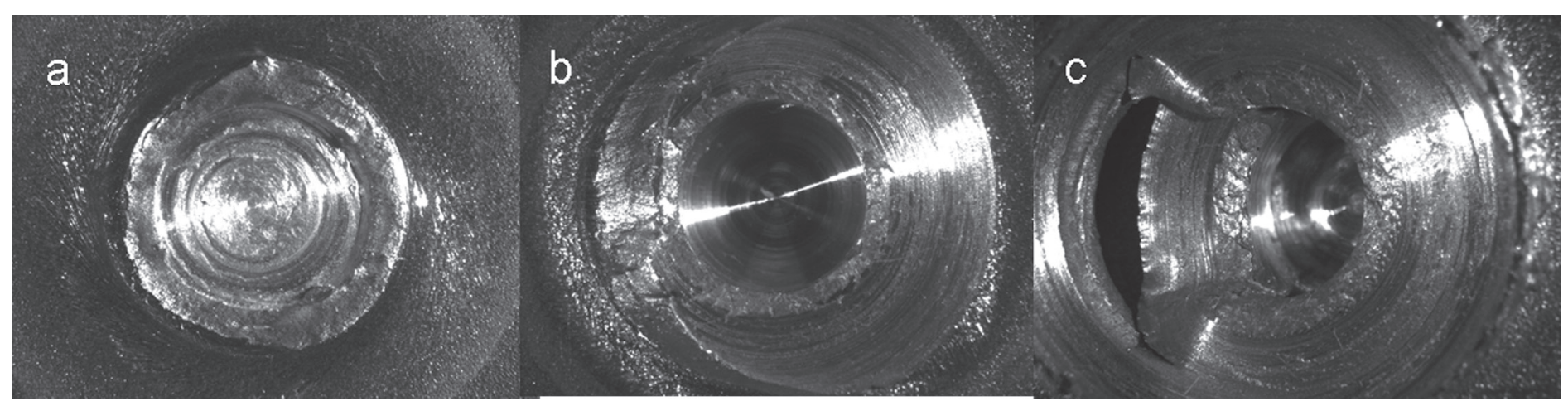

Figura 8- Aspecto dos três tipos possíveis de fratura: a) Falha por cisalhamento b) Falha por arrancamento e c) falha por arrancamento com destacamento da chapa inferior.

No gráfico acima pode ser observado que na maioria dos casos os maiores valores obtidos de resistência ao cisalhamento foram obtidos com os maiores comprimentos de camada de ligação. A utilização de maiores velocidades de rotação também implicou em soldas mais resistentes ao cisalhamento. Provavelmente estes dois fatos estão interligados, ou seja, a utilização de maiores velocidades de rotação implica na produção de soldas com maiores camadas de ligação. Uma possibilidade é que a revolução da ferramenta proporciona a repulsão de material em direções axiais a ferramenta.

\section{FLUXO DE MATERIAL}

De acordo com Ramos [25,26] o fluxo de material no processo de SPFMM segue dois padrões. Um padrão interno próximo a periferia do pino, onde o material é levado da chapa superior a chapa inferior através de um fluxo em helicoidal. E outro padrão externo, onde o material é movido da chapa inferior para a chapa superior após encontrar a resistência da chapa de apoio. Este fluxo segue pela região externa retornando como fluxo interno após encontrar a resistência oferecida pelo material pressionado pelo ombro da ferramenta e consolidando a zona de mistura. A Figura 10 mostra um desenho esquemático de contato, a utilização de maiores velocidades aumenta o comprimento da CL. E a seta indica o possível caminho que uma eventual fratura do corpo de prova seguiria.

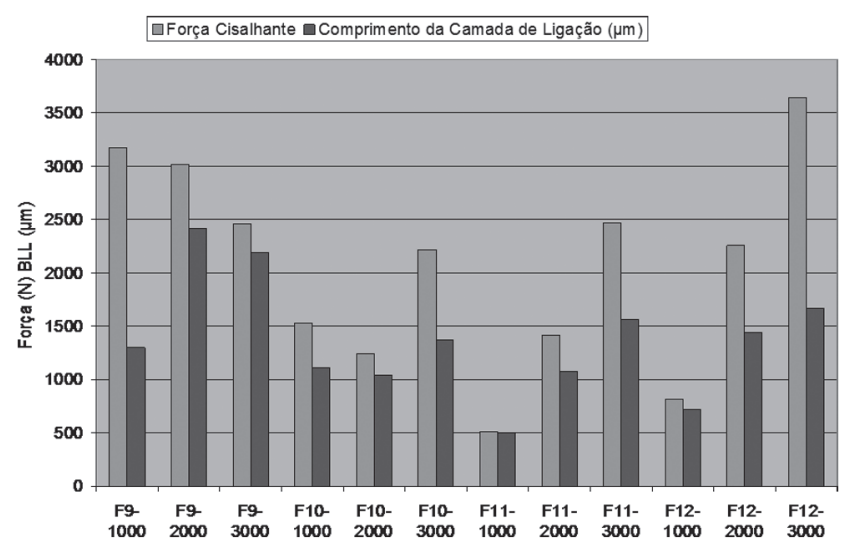

Figura 9- A figura apresenta um gráfico com os valores de resistência ao cisalhamento e comprimentos de camada de ligação para as diferentes combinações de ferramenta com velocidade de rotação. 


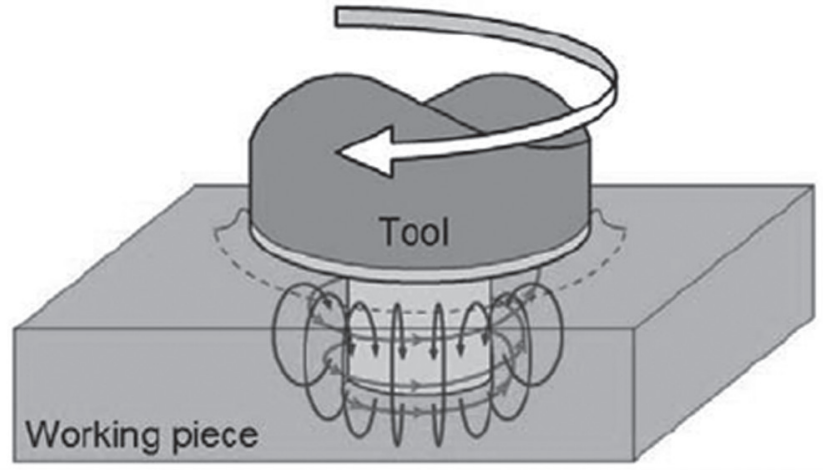

Figura 10- Desenho esquemático mostrando os padrões de fluxo interno (em helicoidal) e o padrão externo (contornando o helicoidal).

A comparação da solda realizada com a ferramenta F12 utilizando a rotação de 1000 RPM e a de 3000 RPM nos mostra que, efetivamente, a camada de ligação é maior, a deformação da fratura é maior, corroborando com a premissa de que a velocidade de rotação promove este tipo de alteração no fluxo de material. Como pode ser observado na Figura 12.

Para as amostras soldadas com a chapa de cobre no interior da junta como traçador, a quantidade de cobre presente nas regiões foi utilizada como indicativo de fluxo de material levado para a chapa superior ou chapa inferior. Nas amostras soldadas com as ferramentas F9 (Figura 13) e F12 (Figura 14), pode ser observada uma maior quantidade de cobre na região a frente da ponta do pino. Isto se deve à geometria do pino, que favorece o aprisionamento de cobre nestas regiões.

As amostras soldadas com o cobre não apresentaram boa qualidade na solda, porém, a motivação é investigar o fluxo do material, portanto não será levada em conta a qualidade da solda. As amostras soldadas com as ferramentas F10 (Figura 15) e F11 (Figura 16), que tem um perfil mais agudo, apresentaram menos cobre na região a frente na ponta da ferramenta. Estas ferramentas aparentemente promoveram uma menor mistura do material, pois a chapa de cobre foi menos alterada quando se utilizou este tipo de ferramenta.

Uma particularidade observada na amostra soldada com a ferramenta F12 é o comportamento semelhante ao mostrado anteriormente na Figura 11, onde ocorre o fluxo de alumínio conforme é apontado para o padrão interno de fluxo sobre um fluxo externo de cobre, o que garantiria um ancoramento mecânico e uma boa mistura de material. Na Figura 17 podemos verificar este fenômeno, que não ficou claro para as amostras soldadas com as outras ferramentas.

A utilização de cobre como traçador é bastante eficiente, devido ao bom contraste existente entre cobre e alumínio, e podemos ter uma boa idéia do fluxo de material. Arbegast [15] obteve resultados semelhantes utilizando pó de cobre como material traçador, Gerlich [17] utilizou o contraste entre o Mg e $\mathrm{Al}$ após o ataque químico para caracterizar o fluxo de material. Em ambos os casos, pode-se perceber a formação do padrão interno (material arrastado pela ferramenta) e do padrão externo (material espremido contra a base que retorna através do fluxo externo). Podemos comparar o formato do cobre armazenado à frente da ferramenta com a zona de mistura de um material soldado com o mesmo pino. A Figura 18 compara os resultados obtidos para amostras soldadas com o pino da ferramenta F10 em uma amostra para visualização do fluxo e para uma amostra para metalografia.
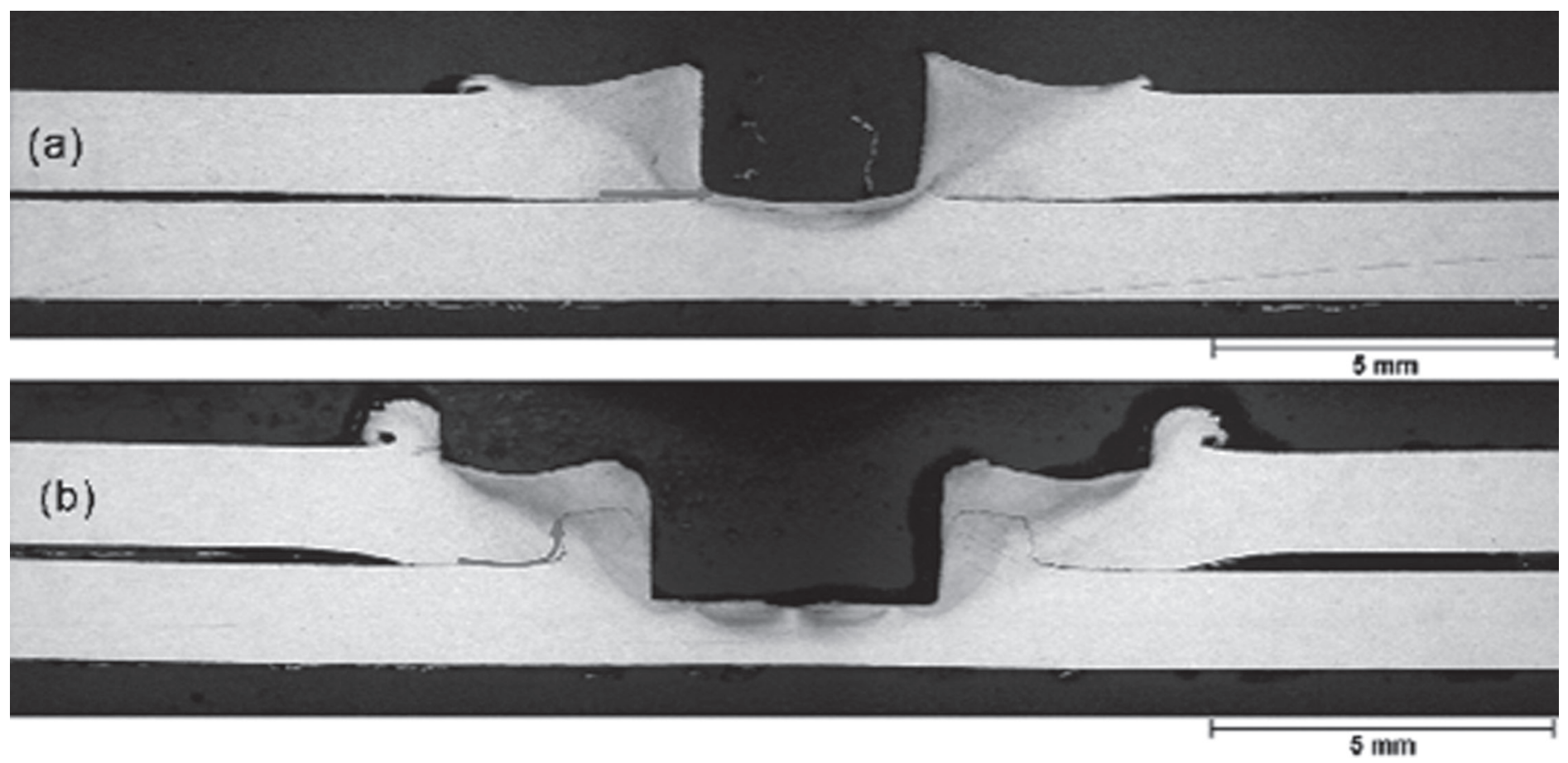

Figura 11- Amostra soldada com menor velocidade de rotação (a) onde há uma menor $\mathrm{Cl}$ e a linha de contato não é modificada. E amostra soldada com maior velocidade de rotação (b) onde se observa uma maior camada de ligação e o deslocamento da linha de contato para cima [26]. 


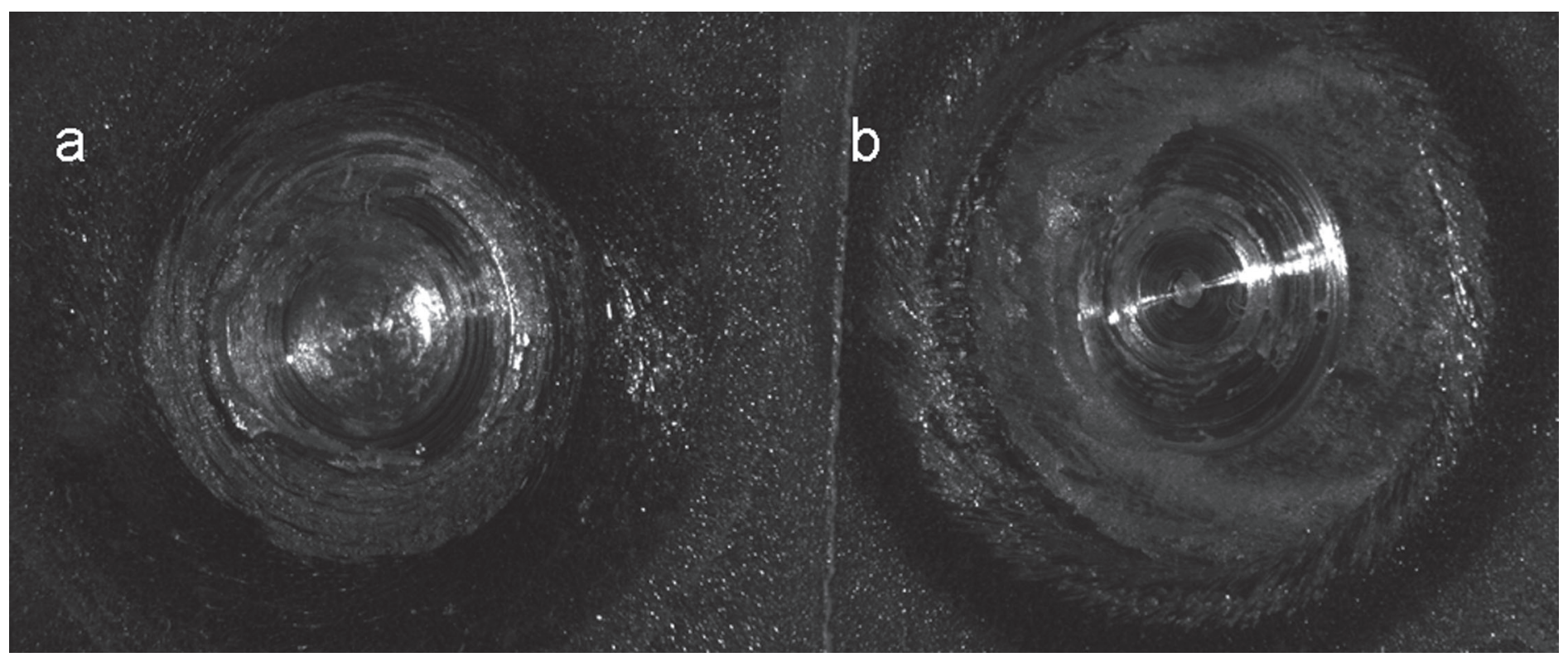

Figura 12- Fractografia das amostras soldadas com ferramenta F12 com: (a) 1000 RPM, mostrando pouca ligação e deformação e (b) 3000 RPM, mostrando alta deformação plástica e comprimento de CL.

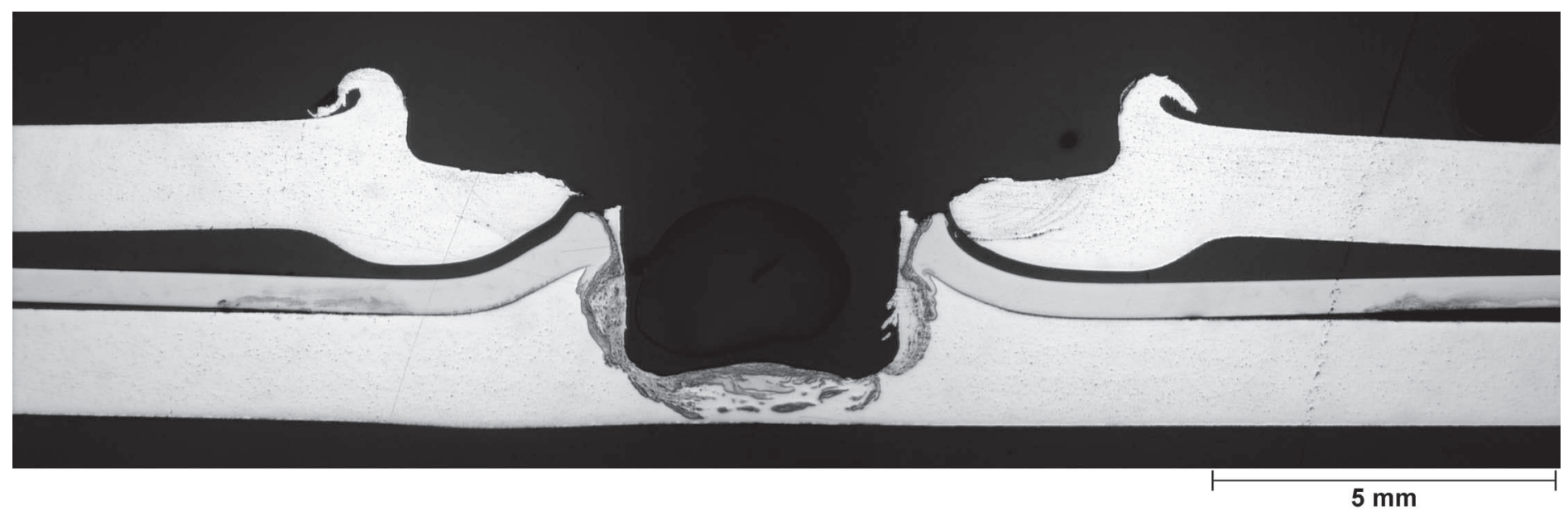

Figura 14- Macro mostrando o resultado obtido para a amostra soldada com a ferramenta F12.

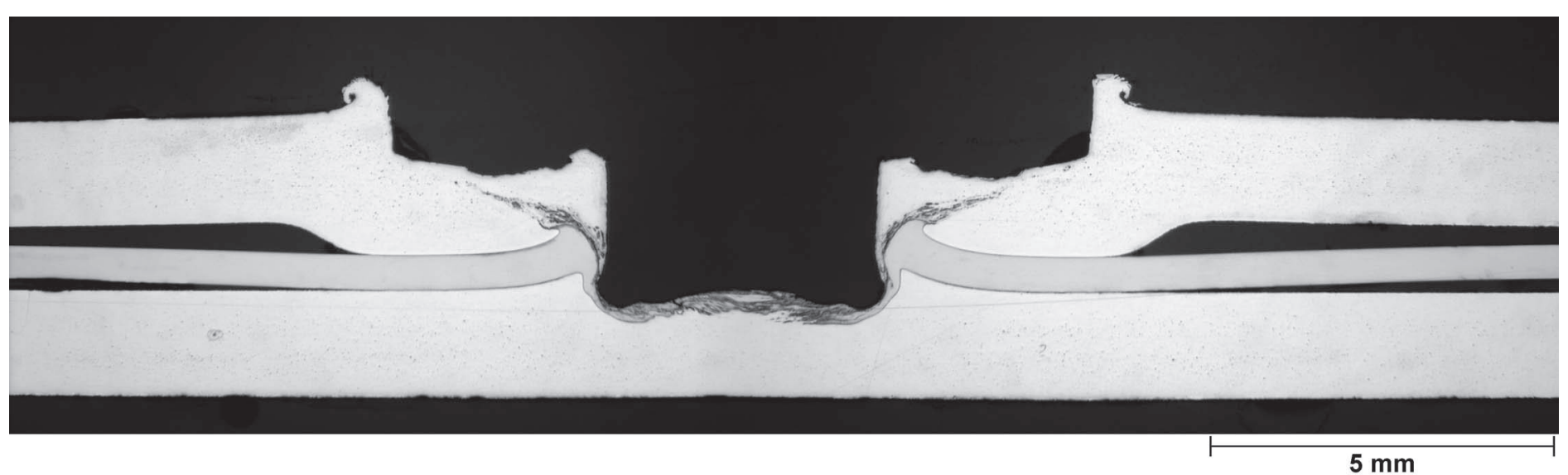

Figura 15- Macro mostrando o resultado obtido para a amostra soldada com a ferramenta F10. 


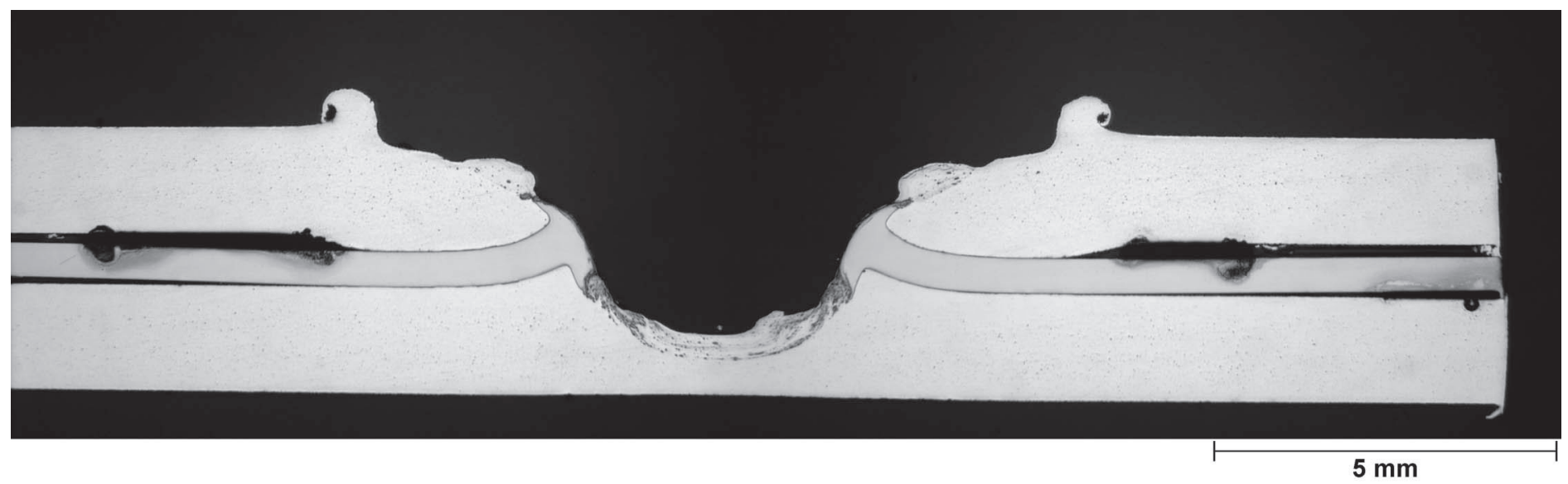

Figura 16- Macro mostrando o resultado obtido para a amostra soldada com a ferramenta F11.

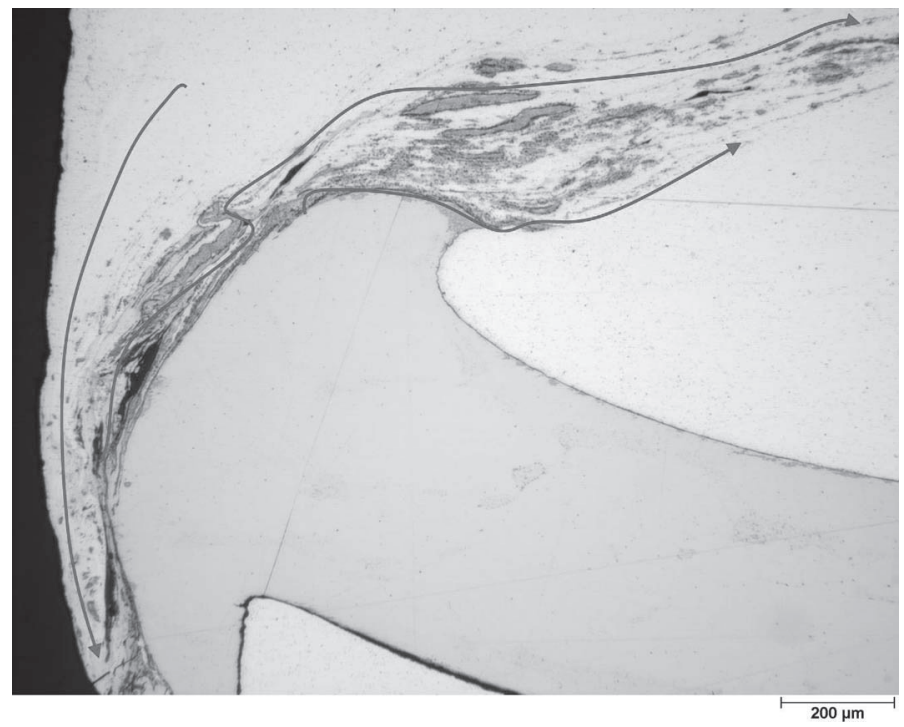

Figura 17- Imagem mostrando o fluxo de alumínio e cobre em detalhe na amostra soldada com a ferramenta F12. As setas dão um indício de sentido de fluxo do material.

\section{MONITORAMENTO DE TORQUE E FORÇAS}

A monitoração do torque e das forças durante o processo foi realizado pela própria máquina. Para análise das forças envolvidas no processo foram encontrados dois picos, um correspondente ao início da penetração do pino nas chapas e outro correspondente ao contato do ombro com a chapa superior. Logo após o segundo pico a força decresce até atingir um patamar que corresponde ao tempo de espera. O tempo de espera é o tempo em que a ferramenta está totalmente penetrada em rotação na junta. Os valores de torque sobem gradualmente até atingir um máximo coincidindo com o pico 2 da força. Isto é esperado já que haverá um contato maior da ferramenta com a junta soldada. Após a remoção da ferramenta a força decresce até zero e o processo termina. A Figura 19 mostra as curvas para torque e forças do processo de uma forma geral, enquanto a Figura 20 mostra os valores máximos de torque e força obtidos no processo. Estes resultados de monitoramento de força e torque foram idênticos aos observados por Gerlich e Su onde os picos de força e torque foram observados, assim como o patamar $[12,15,16,18]$.
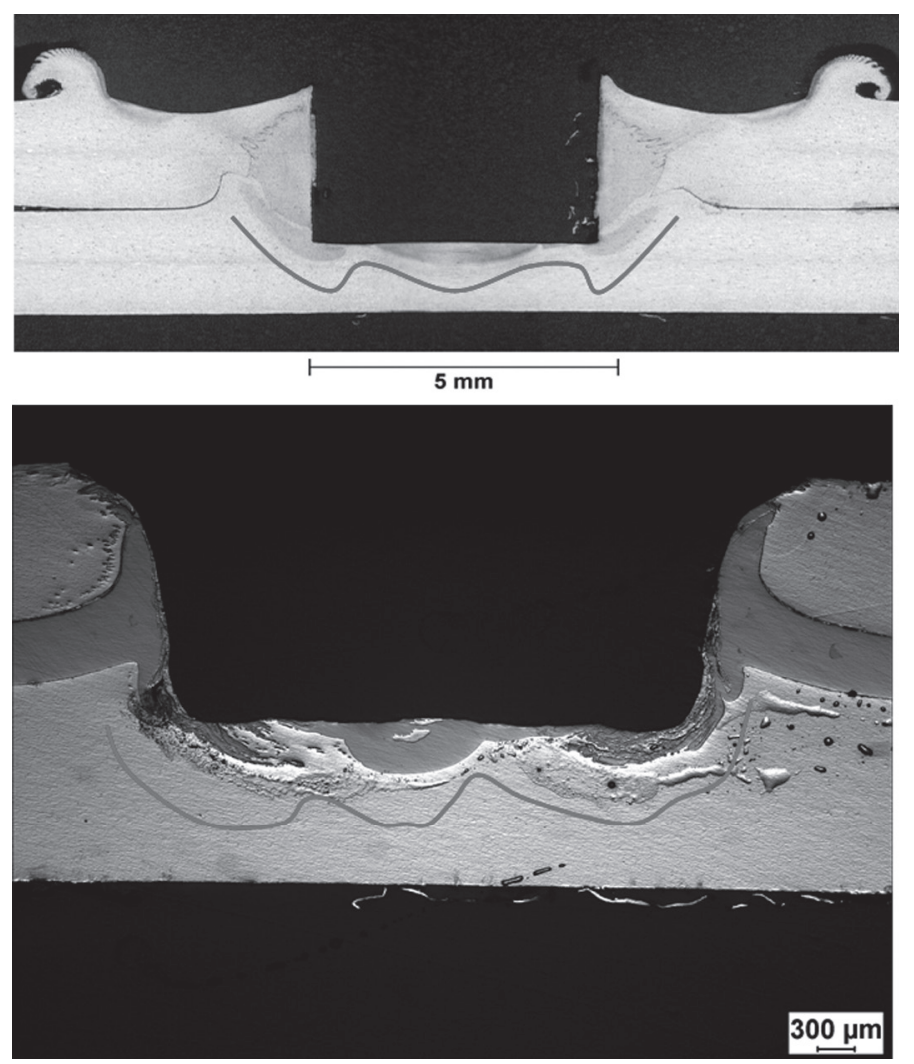

Figura 18- A figura mostra a semelhança entre a região de acúmulo de cobre e a zona de mistura de uma amostra de metalografia. 


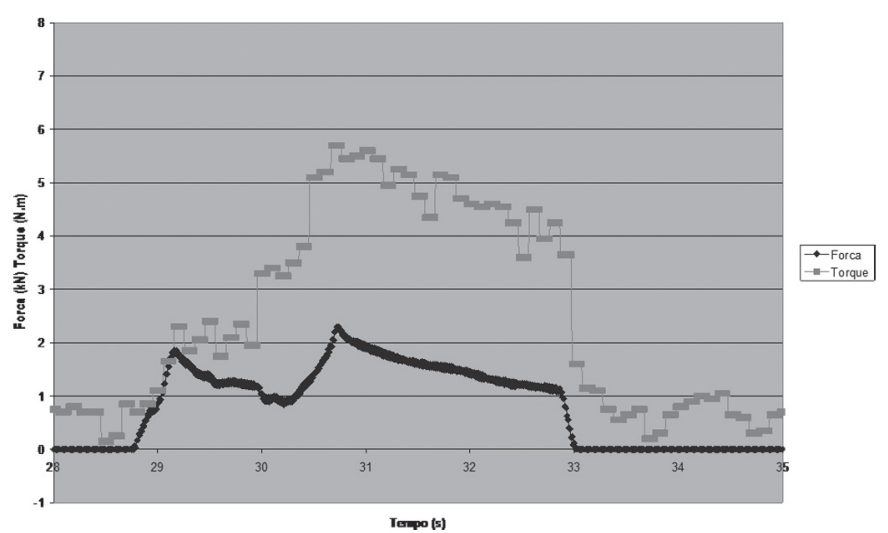

Figura 19- Curvas de força e torque para a amostra soldada com a ferramenta F10@3000 RPM. Onde se observa os picos 1 e 2 da força e a curva de torque atingindo o máximo juntamente com o pico 2 da força.

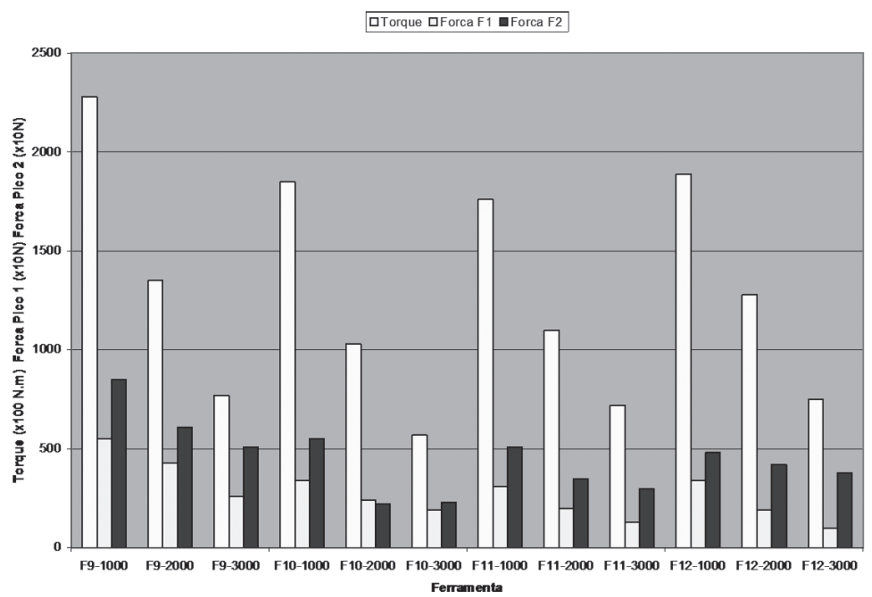

Figura 20- Compilação de valores máximos obtidos em torque e força (picos 1 e 2).

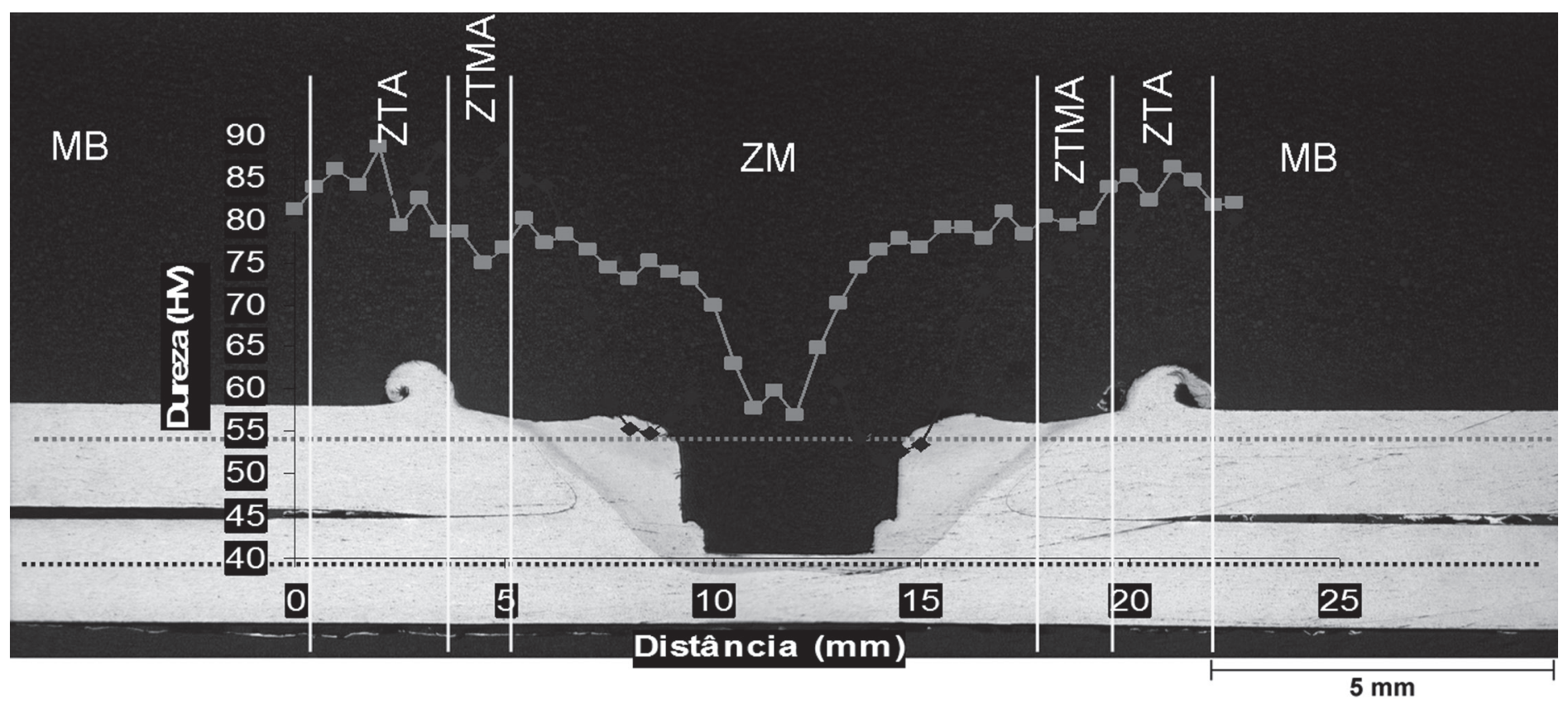

Figura 21- Perfil de microdureza realizado na amostra soldada com a ferramenta F11 com 3000 RPM mostrando as diferentes zonas formadas após o processo de SPFMM.

O perfil de microdureza conseguiu detectar a formação de uma zona afetada pelo calor, o que não fora possível através da metalografia. Os perfis ficaram bastante semelhantes, desta forma será mostrado na Figura 21 um perfil representativo da amostra soldada com a ferramenta F11 com 3000 RPM.

\section{Conclusões}

Pela análise e discussão dos resultados pode-se concluir que:

1- Foi possível realizar a solda da liga utilizando as ferramentas e os parâmetros propostos no trabalho;

2- O perfil da ferramenta demonstrou ter bastante influência sobre o fluxo do material durante a soldagem das amostras;

3- Quanto maior a camada de ligação maior será a resistência mecânica da junta;

4- As soldas realizadas em maiores velocidades de rotação promoveram maior camada de ligação e ainda deslocamento da linha de contato das chapas, favorecendo a fratura de maior energia;

5- A técnica de perfil de microdurezas indicou que existe realmente a formação de uma zona afetada pelo calor;

6- A zona de mistura apresentou um decréscimo de dureza devido à recristalização do material.

7- A melhor condição de soldagem foi utilizando a ferramenta F12, com maior velocidade de rotação, pois foi a condição que combinou maior resistência ao cisalhamento com maior mistura com relação ao fluxo de material. 


\section{Agradecimentos}

Os autores gostariam de agradecer a Capes e ao DAAD, pelo programa PROBRAL, ao HZG e ao LAMEF-UFRGS.

\section{Referências Bibliográficas}

[1] C. Schilling, J.F. dos Santos, Verfahren und vorrichtungzum verbiden von wenigsteins zwei aneinanderliegenden werkstücken nach der mothode des reibrührschweißens, German Patent DE 19955737 B4 2005.11.10. B

[2] C. Schilling and J.F. dos Santos, Method and device for joining at least two adjoining work pieces by friction welding, European Patent EP 1230062 B1, 17.05.2006.

[3] C. Schilling and J.F. dos Santos, Method and device for joining at least two adjoining work pieces by friction welding, United States Patent US 6,722,556 B2, April 20, 2004.

[4] A. Gerlich, P. Su, T.H. North and G. J. Bendzsak, "Friction Stir Spot Welding of Aluminum and Magnesium Alloys", 2005. Materials Forum Volume 29.

[5] C. Schilling, A. v. Strombeck, J.F. dos Santos and N. v Heesen, "A Preliminary Investigation on the Static Properties of Friction Stir Spot Welds. "2nd International Symposium on Friction Stir Welding, June 26-28, 2000, Gothemburg, Sweden. [6] A. Gerlich, P. Su, T. H. North and G. J. Bendzsak, "Mechanism of Stir Zone Formation during Friction Stir Spotwelding". Metallurgical and Materials Transactions. June 2006.

[7] A. Gerlich, P. Su and T.H. North, Peak Temperatures and Microstructures in $\mathrm{Al}$ and $\mathrm{Mg}$ Alloy FSW Spot Welds, Science and Technology of Welding and Joining, December 2004.

[8] A Gerlich, G A Cingara and T H North, "Microstructure and strain rate in Al 7075-T6 spot welds", March 2006, Metallurgical and Materials Transactions A.

[9] Z. Feng, M. L. Santella, S. A. David, R.J. Steel, S. M. Packer, T. Pan, M. Kuo and R.S. Bhatnagar, "Friction Stir Spot Welding of Advanced High-Strength Steels- A Feasibility Study", 2005. SAE international Annual Meeting.

[10] A.C. Addison, A.J. Robelou, Friction Stir Spot Welding: Principal parameters and their effects, Proceedings of the $5^{\text {th }}$ International Symposium on Friction Stir Welding $-5^{\text {th }}$ ISFSW, 14-16 September 2004, Metz, France.

[11] P. Su, A. Gerlich and T. H. North, "Friction Stir Spot Welding of Aluminum and Magnesium Alloys". ". 5th SAE International Annual Meeting, 2005

[12] A. Gerlich, P. Su. T.H North, Tool penetration during friction stir spot welding of $\mathrm{Al}$ and $\mathrm{Mg}$ alloys, Journal of Materials Science 40 (2005 A), p 6473-6481.

[13] T.-Y. Pan, W. Zhu, W. Schwartz. Spot Friction Welding - A New Joining Method for Aluminium Sheets. In: Proceedings of International Automotive Body Congress - IABC 2005, Vol. 2, September 20-21 2005, Ann Arbor, Michigan, 95-99.

[14] H. Badarinarayan, F. Hunt, K. Okamoto. Friction Stir Stitch Welding - Process Development and Mechanical Property Evaluation. 6th International Symposium of Friction Stir Welding ISFSW6, 10-13 October 2006, Montreal, Canada

[15] P. Su, A. Gerlich, T.H. North and G.J. Bendzsak, "Material flow during friction stir spot welding". Sci. Technol. Welding and Joining, August 2005.

[16] Gerlich A, Su P, Yamamoto M and North T. H, Effect of Welding Parameters on the Strain rate and microstructure of Friction Stir Spot Welded 2024 Aluminum Alloy, Journal of Materials Science, 2006.

[17] S. Kalagara and K. H. Muci-küchler, W.J. Arbegast, Preliminary study of material flow in friction stir spot welding using copper as marker material, Friction Stir Welding and Processing IV, TMS 2007, 349-358.

[18] P. Su, A. Gerlich, T.H. North and G.J. Bendzsak, Energy Utilization and Generation During Friction Stir Spot Welding, Science and Technology of Welding and Joining 2006 Vol 11 No 2, 163-169. A

[19] P. Su, A. Gerlich, T.H. North and G.J. Bendzsak, Energy generation and stir zone dimensions in friction stir spot weldings, SAE technical series, 2006, 2006-01-0971. Warrendale, USA. SAE. B

[20] M. Valant, E. Yarraparedddy and R. Kovacevic, A novel tool design for friction stir spot welding, Proceedings of the 7th International Conference on Trends in Welding Research, May 16-20, 2005, Callaway Gardens Resort, Pine Mountain, Georgia, USA.

[21] D. E. Clark, K. S. Miller and C. R. Tolle, "Tool Design in Friction Stir Processing: Dynamic Forces and Material Flow". 7th International Conference of Trends in Welding Research, May 16-20, Pine Mountain, Georgia, USA.

[22] Da Silva, M. Tier, T. Rosendo, F.D. Ramos, C. Mazzaferro, J. Mazzaferro, L. Bergmann, T.R. Strohaecker, J.F. dos Santos, Friction Spot and Friction Stir Spot Welding Processes - A Literature Review, Bulletin of National R\&D Institute for Welding and Material Testing, ISSN 1453-0392, 3/2007.

[23] A. Gerlich, M. Yamamoto and T. North, "Local melting and tool slippage during friction stir spot welding of Alalloys", Journal of Materials Science. Volume 43, pag. 02-11, 2008.

[24]- http://www.mazda.com/publicity/release/2003/02/27 "Mazda Develops World's First Aluminum Joining Technology Using Friction Heat".

[25] Ramos, F.D. Tese de doutorado. PPGE3M. Universidade Federal do Rio Grande do Sul- Escola de EngenhariaDepartamento de Metalurgia, 2008.

[26] RAMOS, F. D. ; M.A.D.Tier ; SILVA, A. A. M. ; MAZZAFERRO, J. ; MAZZAFERRO, C. C. P. ; Rosendo, Tonilson de Souza ; STROHAECKER, T. ; Reguly, Afonso ; dos SANTOS, J. F. . The Influence of the Tool Profile in Friction Stir Spot Welding of the Aluminum Alloy AA6181-T4. TRENDS IN WELDING RESEARCH, v. 8, p. 113-120, 2009. 